\title{
Étude expérimentale des écoulements à bulles turbulents cisaillés
}

\author{
par Michel Lance, Jean-Louis Marié, Elmostafa Moursali, Jean Bataille, \\ du Laboratoire de Mécanique des Fluides et d'Acoustique, Ecole Centrale de Lyon
}

Christian Suzanne, Véronique Roig, Rebei Bel Fdhila, Lucien Masbernat

de l'Institut de Mécanique des Fluides, Toulouse

\section{INTRODUCTION}

Le régime d'écoulement diphasique à bulles se rencontre dans de nombreuses applications industrielles et a été étudié intensivement durant ces dernières années. Cependant, la compréhension des phénomènes physiques impliqués dans ce régime est encore trop limitée pour espérer, à court terme, des prédictions numériques suffisamment précises dans une large gamme de paramètres et de géométries. Un exemple caractéristique est celui des écoulements à bulles en conduites, qui, malgré de nombreux travaux expérimentaux et théoriques (Serizawa, Kataoka, Michiyoshi, 1975), défie encore la simulation numérique. Il semble par conséquent indispensable d'aborder la compréhension des mécanismes physiques régissant l'évolution spatiale et temporelle des écoulements à bulles au travers de l'étude de configurations simples, comme les écoulements cisaillés.

Cet article est une synthèse commune des travaux réalisés sur ce thème par les équipes de l'Institut de Mécanique des Fluides de Toulouse (URA CNRS 5), et du Laboratoire de Mécanique des Fluides et d'Acoustique de Lyon (URA 263), en grande partie dans le cadre du GDR CNRS 1027. Les expériences présentées ici concernent quatre configurations de base : 1) un cisaillement turbulent homogène, 2) une couche de cisaillement plane, 3) une couche limite sur une plaque plane, 4) un élargissement brusque en conduite. Les géométries 1 et 3 ont été étudiées par le groupe de Lyon et les situations 2 et 4 par le groupe de Toulouse. Mis à part le fait qu'ils ont été intensivement décrits en monophasique, ces écoulements «canoniques» permettent d'isoler plusieurs mécanismes d'interaction entre les phases : effet des bulles sur les profils moyens de vitesse du liquide, effet sur la turbulence de la phase continue, distribution spatiale du gaz, capture des bulles par les structures cohérentes à grande échelle. Le domaine de paramètres étudié ici, très voisin pour toutes les géométries étudiées, est le suivant:

- Le diamètre des bulles $d_{B}$ est compris entre 2 et $5 \mathrm{~mm}$, de sorte qu'elles soient plus grande que l'échelle de Kolmogorov, et plus petites que l'échelle intégrale de longueur: $d_{B} / \eta>1, d_{B} / L<1$.

- Le nombre de Reynolds fondé sur la vitesse relative $U_{R}$ est élevé : $U_{R} d_{B} / v_{L}>100$.

- Les fluctuations turbulentes sont le plus souvent petites devant la vitesse terminale des bulles (assimilée ici à $\left.U_{R}\right): u^{\prime} / U_{R}<1$.

- Le taux de vide local est de l'ordre de quelques pourcents $(\alpha<10 \%)$.

- Mis à part certaines régions particulières de l'écoulement, l'intensité $S$ du cisaillement moyen vu par les bulles reste faible $\left(S d_{B} / U_{R}<1\right)$.

Dans ces conditions, la force exercée par les bulles sur le liquide est significative. De plus, la dispersion des bulles par la turbulence est dominée par les structures de taille supérieure au diamètre des bulles. Enfin, le mouvement relatif entre les deux phases est important, de sorte qu' une part importante de l'énergie cinétique fluctuante de la phase continue est imputable aux perturbations de vitesse induites par les bulles (pseudo-turbulence). Ce domaine de paramètres correspond à celui observé dans de nombreuses situations pratiques. Il faut noter que les mécanismes physiques mis en cuvre diffèrent du cas des petites particules ou des microbulles, qui ont été récemment étudiées en détail expérimentalement et numériquement (Wang et Maxey, 1993, Lazaro et Lasheras, 1989, Squires et Eaton, 1991). Les expériences décrites ici sont décrites dans les références suivantes : Lance et al., 1991 ; Roig V., 1993; Moursali et al., 1995 ; Bel Fdhila et al., 1992. Le lecteur pourra s'y référer pour obtenir de plus amples détails sur les montages expérimentaux et les techniques de mesure 


\section{II 圆 CISAILLEMENT HOMOGENE}

Le cas le plus simple d'écoulement cisaillé correspond à un gradient de vitesse moyenne constant, associé à une distribution uniforme du tenseur de Reynolds. Cette géométrie idéale a été explorée en détail dans le cas monophasique (Champagne et al., 1970) et s'avère particulièrement utile pour caractériser l'interaction entre champs moyens et fluctuants. On décrit dans cette section les résultats obtenus pour deux configurations d'écoulements homogènes : le cisaillement linéaire et la déformation pure plane.

\subsection{Installation expérimentale}

Le cisaillement linéaire de vitesse est produit à l'aide d'un système très semblable à celui utilisé par Champagne et al. (1970). Le générateur de cisaillement consiste en une section comportant dix canaux parallèles d'égale largeur, à l'entrée desquels une perte de charge variable est imposée. La veine d'essai qui fait suite à ce générateur est un canal vertical de $2 \mathrm{~m}$ de long et de section carrée de $0,4 \mathrm{~m}$ de côté. Une description plus complète est donnée dans Lance et al., 1991. Le taux de vide étudié est compris entre 0 et $5 \%$, le gradient de vitesse moyenne maximum étant de $3 s^{-1}$, ce qui correspond à une vitesse au centre du canal de $0,5 \mathrm{~m} / \mathrm{s}$. Le dispositif expérimental adopté pour la déformation pure plane est très différent. Le gradient de vitesse est obtenu ici par le biais d'un conduit distordant, de section constante, et dont la forme est définie, classiquement, par le raccordement de la section d'entrée carrée à une section de sortie rectangulaire à l'aide de parois de forme exponentielle. L'intensité de la déformation qui est fixée par le rapport d'aspect de la section de sorttie (égal à 2) vaut ici $1 \mathrm{~s}^{-1}$. Dans les deux expériences, les profils transversaux de taux de vide sont uniformes, afin d'éviter une possible influence des gradients de a sur la production de turbulence.

\subsection{Résultats expérimentaux}

On considère d'abord l'évolution longitudinale du tenseur de Reynolds dans le cas du cisaillement linéaire de vitesse. En l'absence de bulles, l'existence du gradient de vitesse se traduit par l'augmentation de l'énergie turbulente longitudinale $u_{L}^{2}=\overline{u_{L}^{2}}$ (la barre horizontale désigne ici la moyenne locale de phase et l'indice $L$ la phase liquide). Les composantes non alimentées par le champ moyen présentent un déclin, alors que la corrélation croisée - $u v$ tend vers une valeur asymptotique. Ce comportement s'avère profondément modifié en présence de bulles, comme on peut le constater sur la figure 1 . Il apparaît que l'anisotropie entre composantes longitudinales et transversales est considérablement réduite par l'injection de bulles d'air. La situation n'est pas toutefois isotrope dans la mesure ou la tension de Reynolds - w ne s'annule pas. L'explication de cette tendance réside dans une compétition entre la distorsion de la turblence par le champ moyen et les déformations aléatoires des structures turbulentes induites par les champs de vitesse autour des bulles. (qui donnent lieu à la pseudo-turbulence).

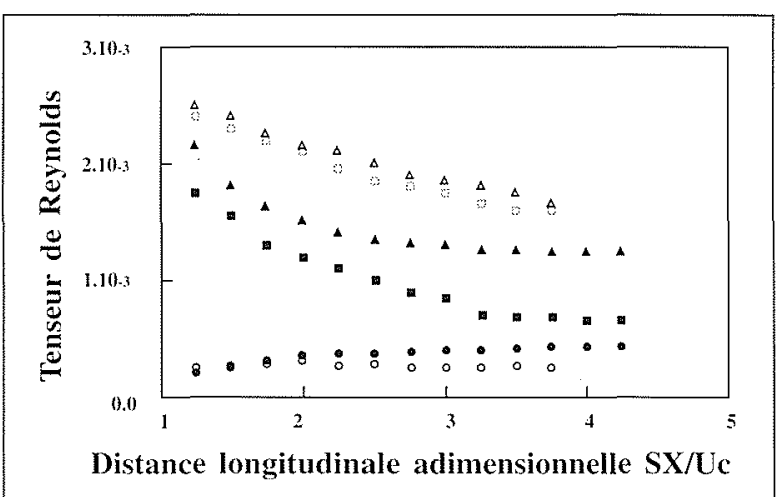

1. Evolution Iongitudinale du tenseur de Reynolds dans un cisaillement linéaire. Intensité du cisaillement $S=2,4 \mathrm{~s}^{-1}$. Vitesse au centre $U_{c}=1 \mathrm{~m} / \mathrm{s}$. Les symboles noirs correspondent à $\alpha=0$, les blancs à $\alpha=0.024$. Triangles : $\overline{u_{L}^{2}} / U_{c}^{2} ;$ carrés : $\overline{v_{L}^{2}} / U_{c}^{2} ;$ cercles : $-\bar{u}_{L} v_{L} / U_{c}^{2}$

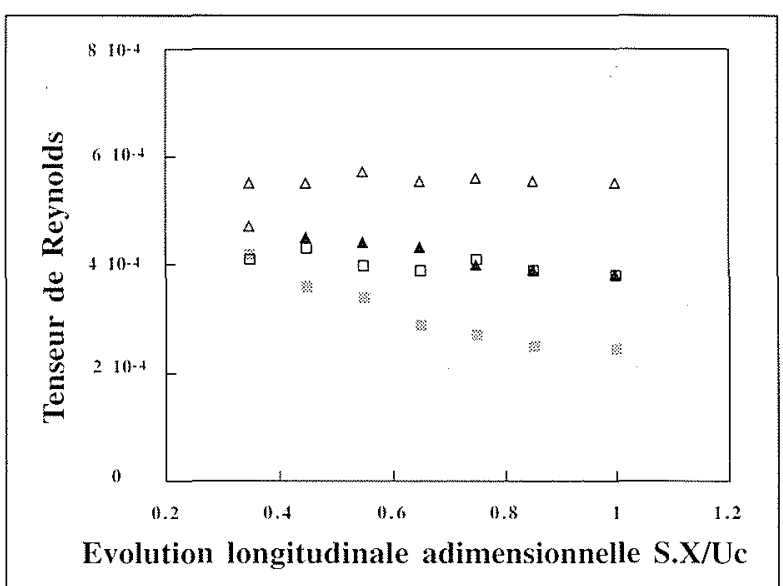

2. Evolution longitudinale du tenseur de Reynolds dans une déformation pure plane. Intensité de la déformation $S=1 \mathrm{~s}^{-1}$. Vitesse au centre $U_{c}=1 \mathrm{~m} / \mathrm{s}$. Les symboles noirs correspondent à $\alpha=0$, les blancs à $\alpha=0.01$. Triangles : $\overline{u_{L}^{2}} / U_{c}^{2} ;$ carrés $: \overline{v_{L}^{2}} / U_{c}^{2}$.

Il est intéressant de remarquer que pour les faibles valeurs du taux de vide, on peut estimer le tenseur de Reynolds par la somme de deux contributions: la première est associée aux fuctuations induites par les bulles, la seconde à la turbulence induite par le cisaillement en l'absence de bulles. Une telle décomposition est toutefois limitée par l'interaction entre les tourbillons turbulents et la pseudo-turbulence, décrite plus haut et responsable de la diminution de l'anisotropie.

Ce comportement du tenseur de Reynolds est confirmé par l'expérience sur la déformation pure plane. Dans ce cas, l'effet de la déformation, qui est perpendiculaire à l'écoulement moyen, est d'amplifier les composantes transversales du tenseur de Reynolds en l'absence de bulles. Ici encore, on observe que l'injection des bulles d'air diminue la production effective de turbulence et réduit l'anisotropie de l'écoulement (fig. 2). 


\section{COUCHE DE MÉLANGE TURBULENTE}

Cette partie est consacrée à l'analyse expérimentale de l'écoulement turbulent air-eau à bulles dans une couche de mélange verticale plane. Comme il a été déjà mentionné, la taille des bulles est plus grande que l'échelle de longueur de Kolmogorov. On peut alors s'attendre à ce que le mouvement relatif des bulles modifie fortement le champ de turbulence de la phase continue.

\subsection{Installation expérimentale et plan d'expérience}

L'installation expérimentale est constituée d'un canal carré vertical de $2 \mathrm{~m}$ de hauteur et de $0,4 \times 0,4 \mathrm{~m}^{2}$ de section transversale dans lequel se développe une couche de mélange ascendante. Deux pompes indépendantes alimentent deux compartiments d'une cuve de tranquillisation située à la base du canal, précédé par un convergent séparée en deux parties indépendantes par une cloison étanche de $2 \mathrm{~mm}$ d'épaisseur. L'injection des bulles d'air se fait à l'entrée du convergent, séparément pour chaque écoulement, par un ensemble de barreaux poreux en inox fritté Les vitesses de chacun des écoulements d'eau peuvent être ajustées entre 0 et $1 \mathrm{~m} / \mathrm{s}$ et le taux de bulles entre 0 et $5 \%$. La vitesse de la phase continue est mesurée par anémométrie à film chaud. Le taux de vide local, la vitesse et la taille des bulles sont obtenus à partir de bisondes optiques à fibre.

Les mesures locales sont réalisées à différentes distances de l'entrée jusqu'à une distance maximale qui assure l'absence d'interaction entre la couche de mélange et les parois latérales de la veine de mesure. Dans la section d'entrée les vitesses de la phase liquide sont uniformes et de valeurs respectives $\overline{U_{L 1}}=0,60 \mathrm{~m} / \mathrm{s}$ et $\overline{U_{L 2}}=0,25 \mathrm{~m} / \mathrm{s}$, soit une différence de vitesse $\Delta \overline{U_{L}}=\overline{U_{L 1}}-\overline{U_{L 2}}$ de l'ordre de $0,35 \mathrm{~m} / \mathrm{s}$. Le nombre de Reynolds caractéristique de la couche de mélange défini par $R e=\Delta \overline{U_{L}} X / v_{L}$ est de l'ordre de $4.10^{5}$ dans la section la plus en aval. La couche de mélange peut être caractérisée par le rapport des vitesses de la phase liquide $\lambda=\Delta \overline{U_{L}} /\left(\overline{U_{L 1}}+\overline{U_{L 2}}\right)$ et par son cisaillement $S$ défini comme le rapport entre la différence de vitesse $\Delta \overline{U_{L}}$ et la largeur conventionnelle de couche de mélange $B_{L}$, il est compris entre 4 et $40 \mathrm{~s}^{-1}$.

\subsection{Discussion des résultats}

\subsubsection{Auto-similitude des profils de vitesses}

Les profils de vitesses moyennes dans la phase liquide en présence et en absence de bulles sont présentés sous la forme adimensionnelle généralement adoptée pour s’as- surer des propriétés d'auto-similitude des couches de mélange en écoulements monophasiques ( $f$ g. 3). On introduit ainsi la vitesse $U^{+}$et la coordonnée adimensionnelles $\eta_{L}$ par:

$$
U^{+}=\frac{\overline{U_{L}}-\overline{U_{L, 2}}}{\overline{U_{L 1}}-\overline{U_{L, 2}}} \quad \eta_{L}=\sigma_{L} \frac{Y-Y_{L 1 / 2}}{X}
$$

$Y_{L 1 / 2}$ désigne la demi-largeur de la couche de mélange et $\sigma_{L}$ est un paramètre sans dimension caractéristique de l'expansion de la zone de mélange.

L'ensemble des mesures monophasiques et diphasiques suivent une loi d'auto-similitude de la forme: $U^{+}=f\left(\eta_{L}\right)$ et sont en bon accord avec la loi analytique proposée par Goertler (Rajaratnam, 1976) :

$$
f\left(\eta_{L}\right)=\frac{1}{2}\left[1+\frac{2}{\sqrt{\pi}} \int_{0}^{\eta_{t}} \exp \left(-y^{2}\right) \mathrm{d} y\right]
$$

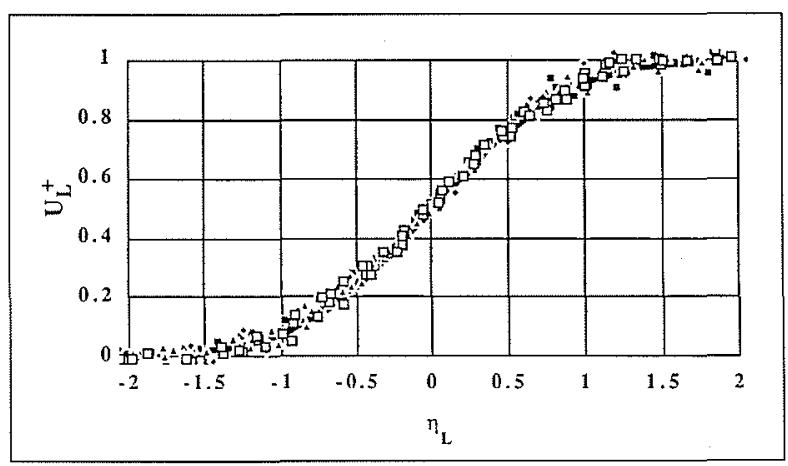

3. Distribution transversale de vitesse moyenne dans la couche de mélange, en monophasique (symboles noirs), et en diphasiques (symboles blancs). Le trait continu est la loi de Goertler.

\subsubsection{Expansion latérale}

Le développement latéral de la couche de mélange peut être caractérisé par la largeur de la couche cisaillée $B_{L}$. En aval d'une zone de transition, $B_{L}$ croît linéairement avec $X$ aussi bien en monophasique qu'en diphasique. Les propriétés d'auto-similitude des couches de mélanges monophasiques sont ainsi résistantes à la présence de bulles dans l'écoulement. Cependant le taux d'expansion de la couche de mélange est fortement amplifié en écoulement à bulles. Pour un même rapport de vitesses $\lambda$, l'expansion de la couche de mélange est deux fois plus importante en écoulement à bulles qu'en l'absence de bulles. Il en résulte que

\begin{tabular}{|c|c|c|c|c|c|c|c|c|}
\hline$\Delta \overline{\mathrm{U}_{\mathrm{L}}}$ & $\mathrm{d}_{\mathrm{B}}$ & $U_{R}$ & $\mathrm{R}_{\mathrm{G}}$ & $S$ & $\lambda$ & $\operatorname{Re}_{B}$ & $\mathrm{We}$ & $\mathrm{Fr}$ \\
\hline $\mathrm{m} / \mathrm{s}$ & $\mathrm{mm}$ & $\mathrm{m} / \mathrm{s}$ & $\%$ & $s^{-1}$ & & & & \\
\hline 0,35 & 3 & 0,30 & 2 & 40 & 0.4 & 900 & 3,9 & 1,7 \\
\hline
\end{tabular}
le paramètre $\sigma_{L}$ de la loi de similitude de vitesse est 
nettement diminué par la présence des bulles. Une analyse détaillée a montré que le développement important de la couche de mélange diphasique peut résulter d'un transfert interfacial de quantité de mouvement associé à la contribution des forces interfaciales moyennes et fluctuantes de masse ajoutée essentiellement.

\subsubsection{Profils de taux de vide}

La présence de la plaque de séparation dans le convergent implique un développement de couche limite de part et d'autre de la plaque. Il en résulte l'apparition d'un pic de taux de vide en aval immédiat de celle-ci. Ce pic décrô̂t en intensité dans l'écoulement, et est dévié latéralement de façon significative vers la partie l'écoulement à faible vitesse. Son évolution se distingue de l'évolution d'un traceur passif dans la mesure où les bulles sont soumises à l'action spécifique des forces moyennes et fluctuantes: d'inertie, de gravité, de trainée et de portance. On peut penser également que l'action des structures cohérentes inhérentes aux couches de mélange puissent avoir une action importante sur la répartition des bulles dans l'écoulement.

\subsubsection{Turbulence dans la phase liquide}

Les fluctuations de vitesses longitudinales de la phase continue sont tracées (fig. 4) sous forme adimensionnelle: $u_{l}^{2+}=\overline{u_{L}^{2}} / \Delta \overline{U_{l}^{2}}$ pour deux expériences.

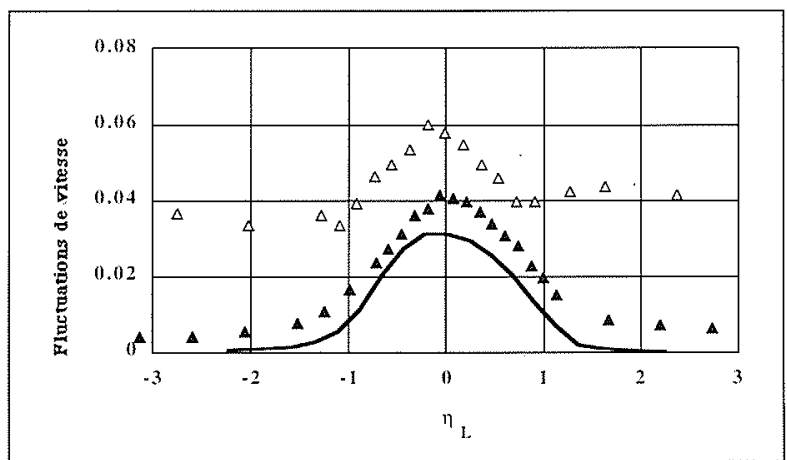

4. Fluctuations de vitesse adimensionnelles dans le liquide $\overline{u_{L}^{2}} /\left(\Delta \overline{U_{L}}\right)^{2}$. Monophasique (\$), écoulement à bulles $(\triangle)$ et données de Wygnanski and Fiedler.

En monophasique les profils de turbulence suivent une répartition d'auto-similitude. En écoulements à bulles, on observe un comportement semblable avec néanmoins un niveau moyen de turbulence nettement plus important. Est-il possible de distinguer la turbulence produite par cisaillement de celle induites par les bulles (pseudoturbulence) afin de quantifier le niveau et la nature de chacune? Suivant Lance and Bataille (1991) dans leur analyse d'écoulement turbulent uniforme à bulles, et sous l'hypothèse que la turbulence de cisaillement et la pseudoturbulence ne sont pas fortement corrélées, on peut supposer que l'énergie turbulente longitudinale mesurée $\overline{u_{L}^{2}}$ représente la somme de l'énergie turbulente longitudinale due au passage des bulles $\overline{u_{B I}^{2}}$ lénergie turbulente longitudinale due au cisaillement $\overline{u_{S l}^{2}}$ dans la couche de mélange. Selon Lance and Bataille (1991) la pseudoturbulence peut être estimée à partir du taux local de vide $a$ et de la vitesse de glissement entre phase $U_{R}$ :

$$
\overline{u_{B I}^{2}} \approx \alpha U_{R}^{2}
$$

Sur la figure 5 , les profils d'intensités turbulentes $\overline{u_{S I}^{2}}$ calculés sont présentés sous forme adimensionnelle. La répartition de cette énergie de fluctuations est proche de la répartition d'énergie turbulente obtenue pour un écoulement monophasique équivalent. Ce résultat semble prouver que même en situation de turbulence inhomogène, il est possible en première approximation d'estimer la turbulence induite par les bulles par une relation analytique simple et de modéliser la turbulence de cisaillement par des concepts classiques de turbulence monophasique.

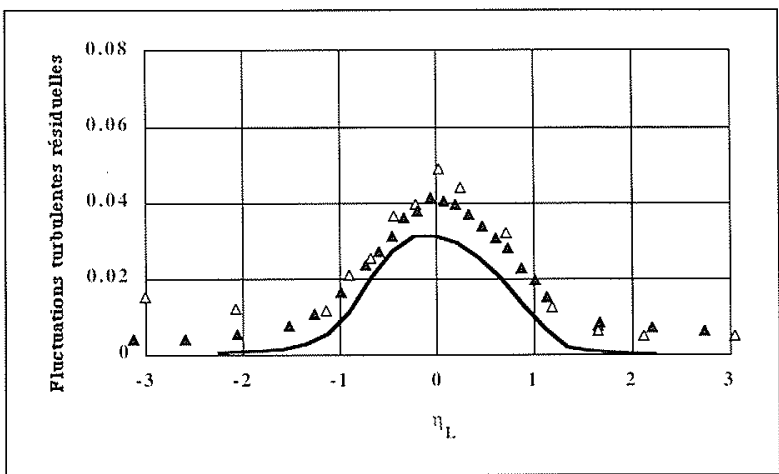

5. Fluctuations de vitesse résiduelles $\left(\overline{u_{L}^{2}}-\alpha U_{R}^{2}\right) /$ $\left(\Delta \overline{U_{L}}\right)^{2}$. Monophasique $(\Delta)$, diphasique $(\triangle)$ and Wygnanski and Fiedler data (trait continu).

Les principaux résultats de cette étude peuvent être résumés comme suit :

- Les profils de vitesses moyennes dans la phase liquide et dans la phase gazeuse conservent les lois d'autosimilitudes des couches de mélange monophasiques. Cependant le taux d'expansion de la couche de mélange est bien plus important que pour les couches de mélange monophasiques : cette amplification résulte des transferts interfaciaux de quantité de mouvement.

- Les fluctuations de vitesses dans la phase continue sont fortement amplifiées par la présence des bulles dans l'écoulement. L'énergie de fluctuations en écoulement dispersé peut être considérée en première approximation comme une simple superposition d'une turbulence de cisaillement et d'une pseudo-turbulence induite par la présence des bulles qui peut être attribuée à un effet local des forces interfaciales et essentiellement de masse ajoutee. 


\section{COUCHE LIMITE TURBULENTE LE LONG D'UNE PLAQUE PLANE VERTI- CALE}

\subsection{Installation expérimentale}

Une description détaillée du dispositif expérimental est donnée dans Lance et Bataille (1991). La section d'essai est un canal vertical, de $2,5 \mathrm{~m}$ de longueur et de section carrée de $0,4 \mathrm{~m}$ de côté. La vitesse moyenne du liquide peut varier entre 0 et $1,5 \mathrm{~m} / \mathrm{s}$. Les bulles d'air sont injectees uniformément à l'aide d'un ensemble de 312 aiguilles de diamètre intérieur égal à $0,4 \mathrm{~mm}$, et fixées sur une grille de turbulence constituée par des barreaux cylindriques de diamètre $8 \mathrm{~mm}$ formant un réseau de maille $40 \mathrm{~mm}$. Le taux de vide ainsi obtenu est uniforme et varie de 0 à $6 \%$. La plaque verticale le long de laquelle se développe la couche limite est placée au centre de la section d'essai. Elle est munie, à son bord d'attaque, d'un ruban rugueux qui déclenche la transition. Les mesures sont effectuées à quatre hauteurs, à partir du début de la plaque : $X=0,07 \mathrm{~m}$, $X=0,2 \mathrm{~m}, X=1 \mathrm{~m}, X=1,5 \mathrm{~m}$. Les mesures monophasiques effectuées à l'aide de la vélocimétrie laser à effet Doppler montrent qu'en l'absence de bulles, la structure de la couche limite est conforme aux données de la littérature. On vérifie en particulier que le gradient de pression longitudinal a un effet négligeable et que la turbulence externe, dont l'intensité est inférieure à $1 \%$, n'affecte pas les profils de vitesse. L'épaisseur de couche limite est typiquement de $22 \mathrm{~mm}$ à $1 \mathrm{~m}$ du bord d'attaque, pour une vitesse extérieure $U_{L E}$ de $\mathrm{I} \mathrm{m} / \mathrm{s}$. Les profils de taux de vide en amont de la plaque, obtenus à l'aide d'une sonde optique, sont uniformes. La granulométrie des bulles présente deux régimes: : pour les petits taux de vide $(0 \%<a<1,5 \%)$, en amont de la plaque, le diamètre des bulles est pratiquement monodisperse autour de $3.5 \mathrm{~mm}$. En revanche, pour les taux de vide plus élevés $(3 \%<a<5.5 \%)$, le diamètre équivalent est compris entre 3 et $8 \mathrm{~mm}$, avec une valeur moyenne de $6 \mathrm{~mm}$.

\subsection{Résultats expérimentaux et discussion}

La figure 7 montre l'évolution des profils de taux de vide dans la section $X=1 \mathrm{~m}$. Ceux-ci montrent l'existence d'un pic d'amplitude $\alpha_{.}$situé à une distance de la plaque de l'ordre du rayon équivalent des bulles. Ces profils indiquent également que les bulles restent séparées de la paroi par une distance approximativement égale à l'épaisseur de la sous-couche visqueuse. Les maxima de taux de vide observés pour cette configuration simple sont semblables à ceux signalés par de nombreux auteurs dans leur étude des écoulements à bulles en conduite verticale (Serizawa et al., 1975). Il a été vérifié que cette inhomogénéité dans la répartition de la phase gazeuse n'était pas une simple conséquence de la décélération de l'écoulement au voisinage du bord d'attaque. Ce dernier effet, quoique signi-

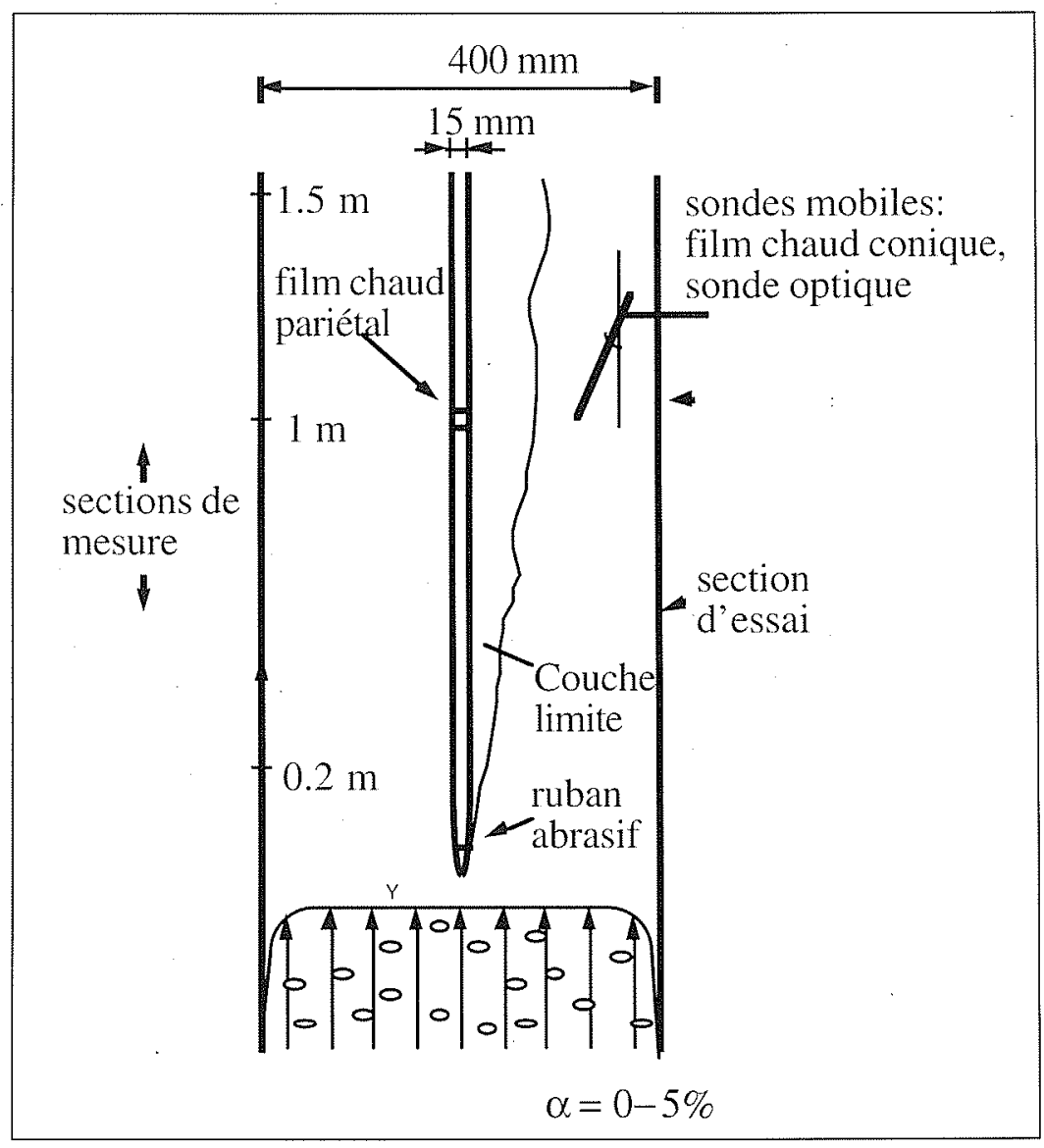

6. Schéma de la section d'essai. 


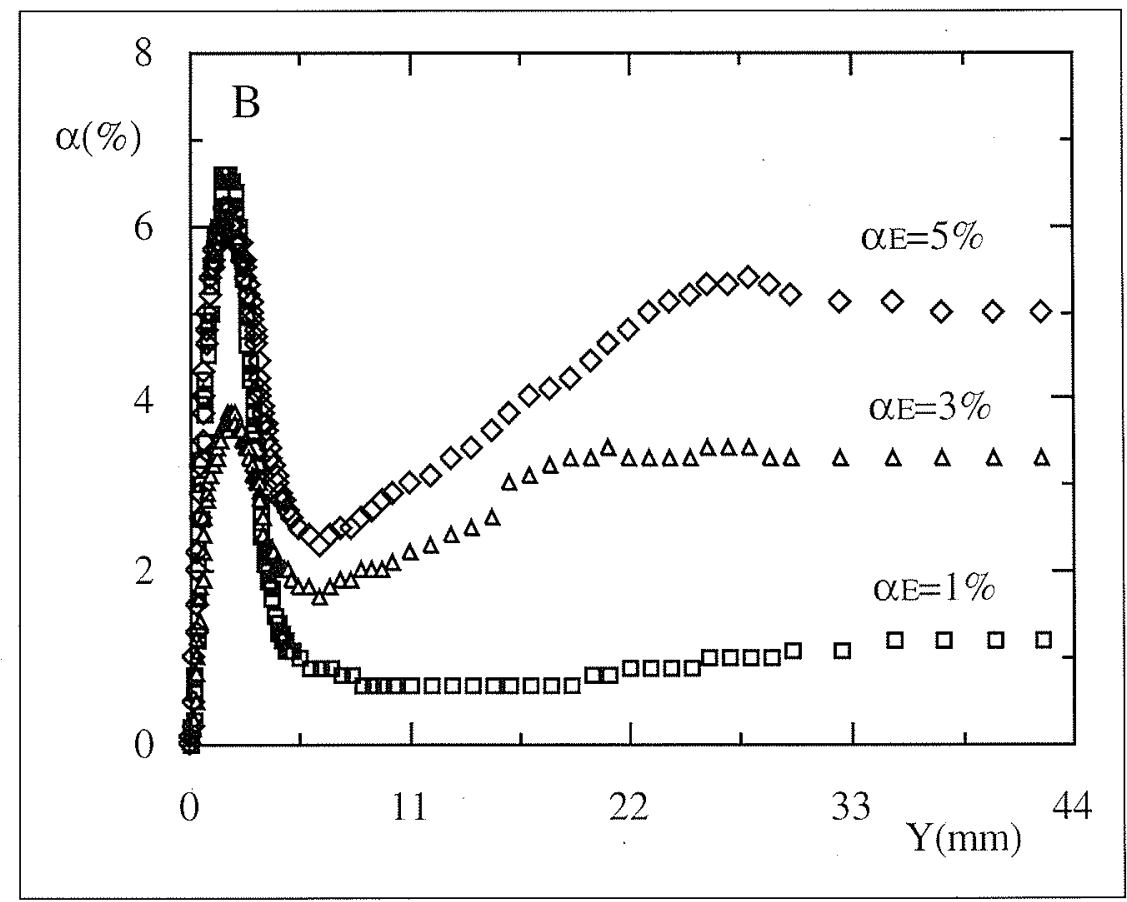

7. Profil de taux de vide à $X=1 \mathrm{~m} ; U_{L E}=1 \mathrm{~m} / \mathrm{s}$.

ficatif, ne peut expliquer à lui seul l'amplitude des pics. Par ailleurs, des visualisations par vidéo rapide permettent d'observer un nombre important de déflections de bulles vers la paroi. Ces migrations semblent aléatoires et caractérisées par des échelles de temps brèves. De plus, il a été constaté que la probabilité de capture d'une bulle à la paroi dépend de son diamètre: les bulles de diamètre $3,5 \mathrm{~mm}$ migrent plus facilement que les bulles de diamètre plus élevé correspondant au second régime mentionné plus haut. Des conclusions similaires ont été faites par Serizawa et al. (1988), Monji \& Matsui (1991), Liu (1993), et Zun et al. (1992). Il n'est pas vraiment surprenant que le diamètre des bulles joue un rôle important. En effet, bien que ceci ne soit pas encore bien éclairci, il est raisonnable d'associer la déflection des bulles dans un écoulement fortement cisaillé turbulent aux mécanismes suivants :

- Déformation de la bulle et modification de son sillage par le gradient de vitesse,

- Interaction entre la bulle et son sillage avec les structures turbulentes environnantes.

Les deux phénomènes sont partiellement contrôlés par le diamètre $d_{B}$. En complément de l'étude par vidéo rapide, le phénomène de migration a été plus quantitativement caractérisé grâce à une sonde à fibre optique, qui permet d'accéder au nombre de bulles $N_{B}$ par unité de temps migrant entre deux sections le long de la plaque plane. Les résultats sont présentés sur la figure $8 . N_{B}$ est porté en fonction du taux de présence local extérieur pour différentes vitesses extemes ULE. Il est adimensionnalisé par la fréquence des éjections turbulentes dans la couche limite turbulente (Blackwelder \& Haritonidis, 1983). La bonne corrélation des données ainsi représentées suggère que la migration des bulles résulte de leur interaction avec les structures turbulentes à l'origine des «bursts».

L'influence de ces pics de taux de vide sur la distribution de vitesse a également été étudiée, à l'aide de sondes

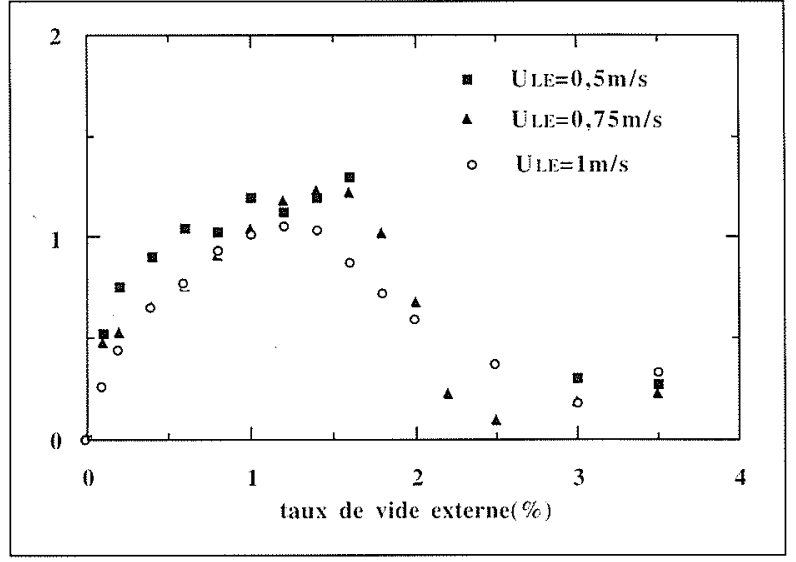

8. Fréquence de migration des bulles adimensionnalisée par la fréquence des éjections turbulentes.

coniques à film chaud miniatures. En particulier, on a représenté sur la figure 9 les profils logarithmiques de vitesse en fonction de la variable intérieure $Y^{+}$. On remarque la persistance de la loi logarithmique, avec toutefois une modification de la constante de Karman et de la constante additive, qui montrent une dépendance envers $\alpha_{E}$ et $U_{L E}$. La figure 10 indique que l'intensité turbulente longitudinale augmente de manière significative du fait de la présence des bulles. Un tel accroissement n'est pas systématique en écoulement en conduite, selon les conditions d'entrée, une décroissance étant même constatée pour les vitesses élevées (Serizawa et al., 1975, Wang et al., 1987). On observe ici trois zones distinctes sur les profils. Dans la région exterieure $\left(Y_{+}>800\right)$, l'accroissement est proportionnel à $\sqrt{\alpha} U_{R}$, ce qui suggère que la pseudoturbulence est l'effet dominant (Lance et Bataille, 1991). 


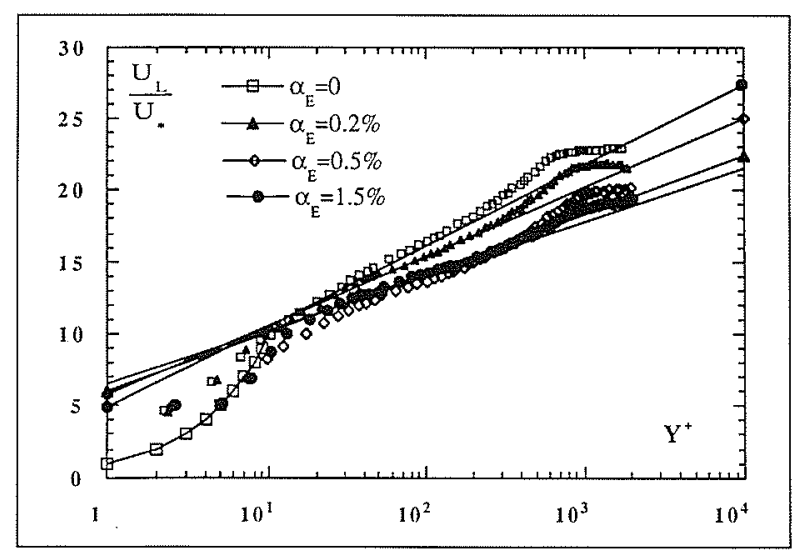

9. Vitesse moyenne du liquide rapportée à la vitesse de frottement, en fonction de la distance adimensionnelle à la paroi. Vitesse moyenne extérieure à la couche limite: $U_{L E}=1 \mathrm{~m} / \mathrm{s} . X=1 \mathrm{~m}$ du bord d'attaque.

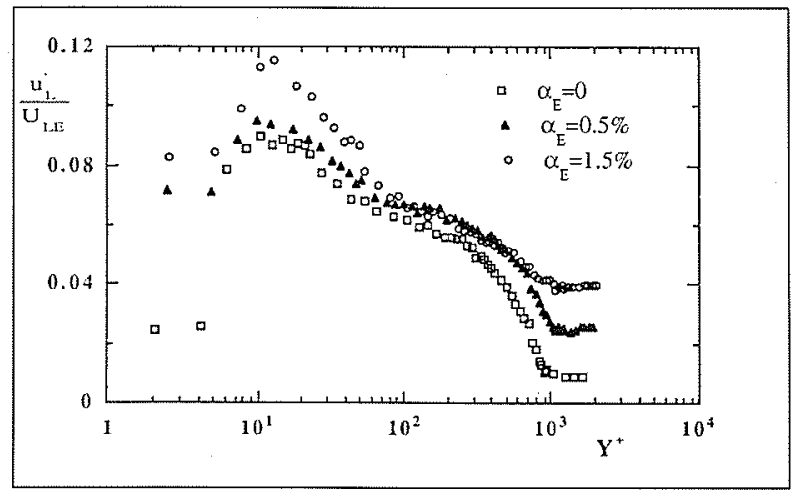

10. Intensité turbulente longitudinale en fonction de la distance adimensionnelle à la paroi pour plusieurs valeurs du taux de vide extérieur. $U_{L E}=1 \mathrm{~m} / \mathrm{s}$. $X=1 \mathrm{~m}$ du bord d'attaque.

Près de la paroi ( $Y_{+}=10$ à 30), l'intensité est proportionnelle à la vitesse de frottement $U_{+}^{\prime}$ ce qui indique une augmentation de la production à la paroi, associée à un gradient de vitesse plus élevé du fait de la présence des bulles d'air le long de la paroi. En revanche, il est plus surprenant de constater une très faible modification dans la région intermédiaire $(30<Y+<200)$, où le taux de vide est maximum.

\section{V ÉLARGISSEMENT BRUSQUE EN CONDUITE}

\subsection{Moyens expérimentaux}

L'installation expérimentale (fig. II) est constituée par une conduite verticale de $5 \mathrm{~cm}$ de diamètre $\left(D_{1}\right)$ suivie d'une seconde conduite de $10 \mathrm{~cm}$ de diamètre $\left(D_{2}\right)$, la hauteur étant de $12 \mathrm{~m}$. Afin de réaliser des conditions de plein développement de l'écoulement dispersé à bulles, l'expansion brusque est située à $9 \mathrm{~m}$ de la section d'entrée constituée d'un injecteur eau-air.

Les débits d'eau examinés correspondent à des vitesses débitantes comprises entre 2 et $3 \mathrm{~m} / \mathrm{s}$ avant l'élargissement. Les mesures de vitesses moyennes et les différentes composantes du tenseur de Reynolds sont réalisées par ané-

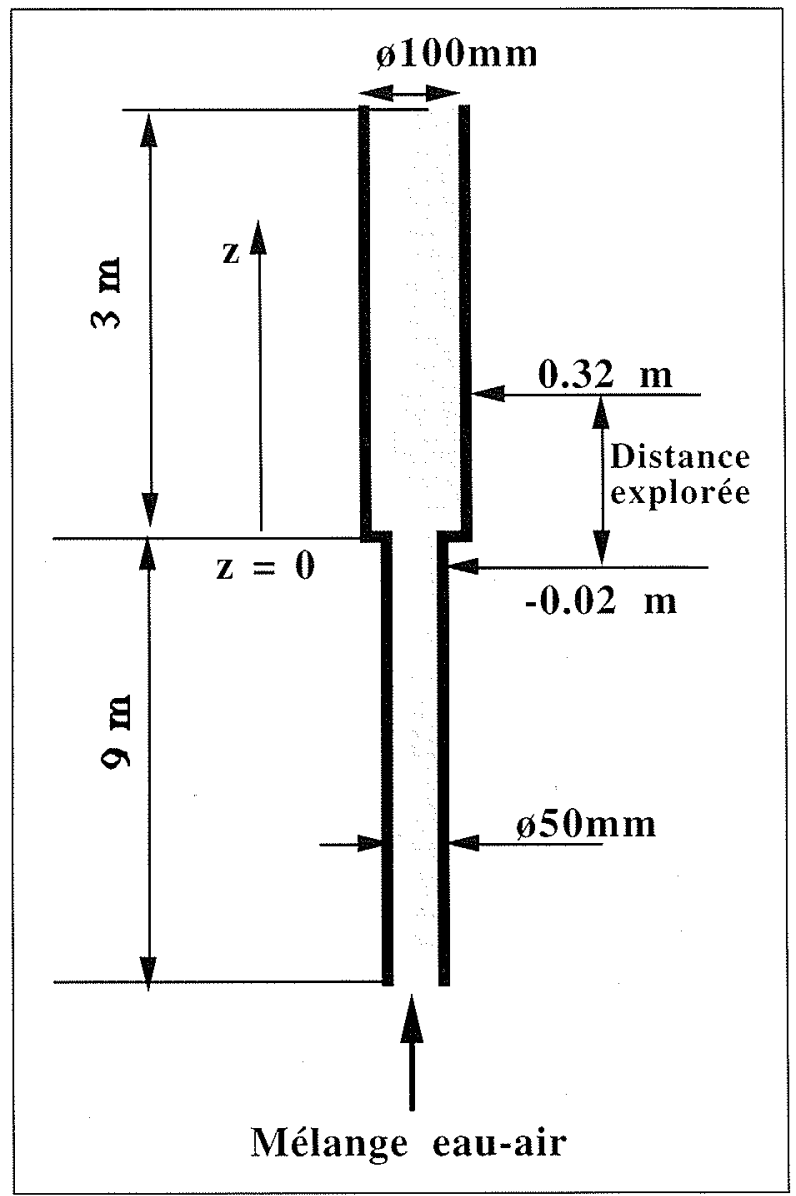

11. Dispositif expérimental pour l'élargissement brusque.

\begin{tabular}{|l|l|l|l|l|l|l|l|l|}
\hline $\mathrm{U}_{\mathrm{L}}$ & $\mathrm{d}_{\mathrm{B}}$ & $\mathrm{U}_{\mathrm{R}}$ & $\mathrm{R}_{\mathrm{G}}$ & $\mathrm{S}$ & $\mathrm{Re}_{\mathrm{L}}$ & $\mathrm{ReB}$ & $\mathrm{We}$ & $\mathrm{Fr}$ \\
$\mathrm{m} / \mathrm{s}$ & $\mathrm{mm}$ & $\mathrm{m} / \mathrm{s}$ & $\%$ & $\mathrm{~s}^{-1}$ & & & & \\
& & & & & & & & \\
2.5 & 2 & 0.25 & 12 & 100 & 120000 & 500 & 1.8 & 3.2 \\
\hline
\end{tabular}


mométrie à film chaud, la vitesse et la taille des bulles sont obtenues à partir de bisondes optiques à fibre. Des mesures locales ont été effectuées dans différentes sections transversales en amont et en aval de l'élargissement. Les principales caractéristiques des écoulements à bulles étudiés sont reportées ci-après, $R_{G}$ représentant le taux de vide moyen dans une section transversale, $S=2 \overline{U_{L}} / D_{1}$ le cisaillement moyen, $R e_{L}=\overline{U_{L}} D_{1} / V_{L}$ le nombre de Reynolds de la phase liquide, $R e_{B}=U_{R} d_{B} / v_{L}$ le nombre de Reynolds associé aux bulles, $W_{e}=\rho_{L} U_{R}^{2} d_{B} / \sigma$ le nombre de Weber et $F r=U_{R} / \sqrt{g d_{B}}$ le nombre de Froude.
L'analyse des profils transversaux de vitesses moyennes, de l'énergie de fluctuation turbulente et des différentes composantes du tenseur de Reynolds obtenus en phase liquide (fig. 12) prouve que qualitativement, la présence des bulles ne modifie pas la structure interne de l'écoulement. Les forts taux de turbulence et de cisaillement des écoulements étudiés, mais surtout le fait que la vitesse de glissement entre phase $U_{R}$ reste faible devant la vitesse débitante de la phase liquide expliquent en grande partie ce résultat. A même débit liquide la présence des bulles réduit la section de passage du liquide et provoque une

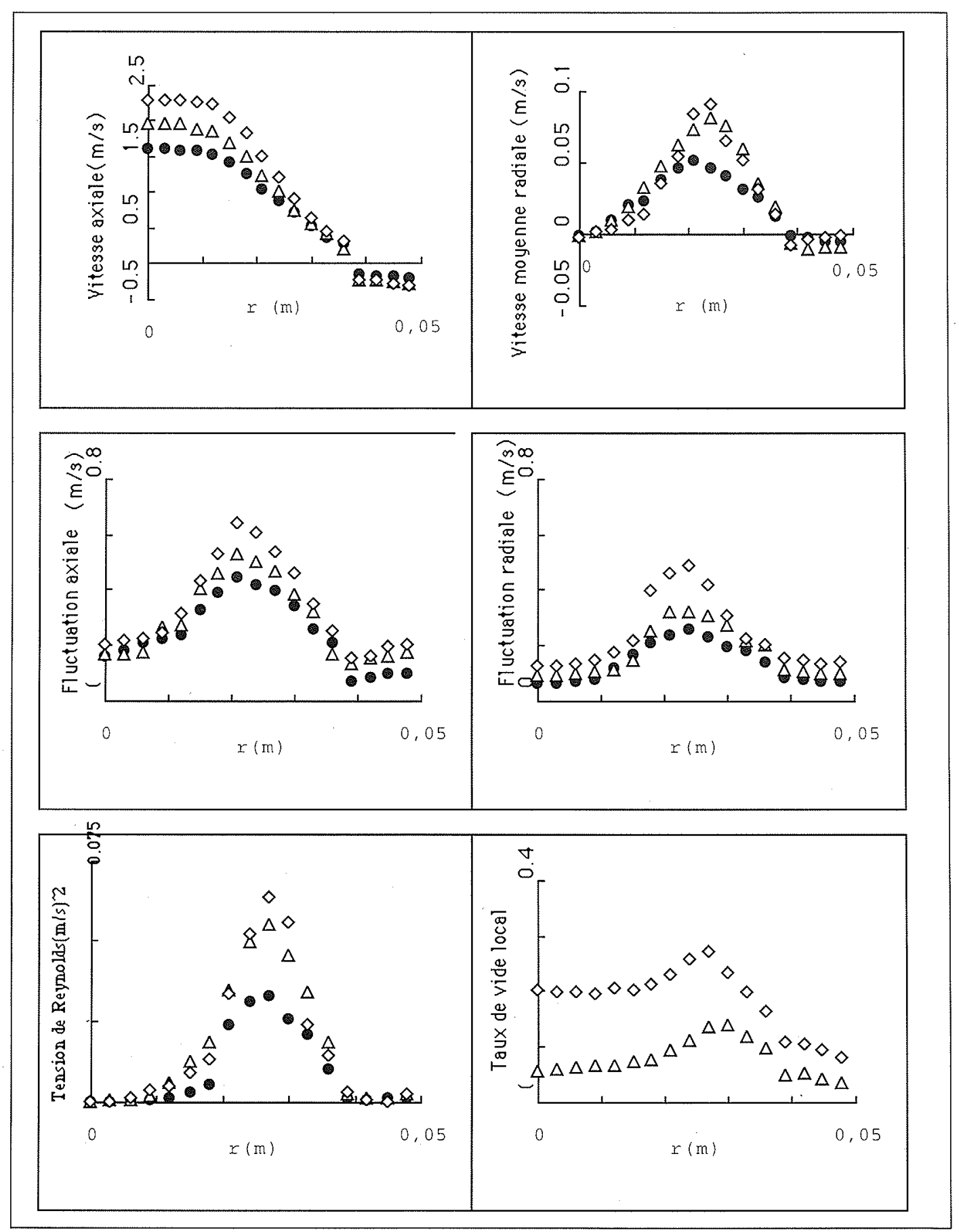

12. Vitesses moyennes, fluctuations turbulentes, tensions de Reynolds et taux de vide à $z=13 \mathrm{~cm}$ en aval de l'élargissement. Monophasique $(\odot)$ et régime à bulles $(\triangle, \diamond)$. 
augmentation sensible de la vitesse de la phase continue. Les profils adimensionnels de vitesses moyennes semblent suivent les mêmes lois d'auto-similitudes en présence et en absence de bulles. Les fuctuations de vitesses longitudinales et radiales de vitesses augmentent également en écoulement à bulles mais gardent des structures semblables à celles obtenues en écoulement monophasique. Cependant les fluctuations de vitesses induites par les bulles (pseudoturbulence) influent plus amplement sur la composante radiale de fluctuation que sur la composante longitudinale, et conduit ainsi à réduire l'anisotropie de la turbulence dans la couche de mélange. Cependant le cisaillement turbulent dans la phase liquide: $-\overline{u_{L} v_{L}}$ est également augmenté. Ainsi, à même débit d'eau la présence des bulles augmente à la fois la vitesse moyenne, les fuctuations longitudinales et radiales de vitesses et le cisaillement et en conséquence la production d'énergie turbulente. La distribution du taux de vide local présente un pic important, jusqu à $38 \%$. Ce pic de bulles créé en proche paroi dans l'écoulement en amont de l'élargissement, décroît progressivement dans la couche de mélange en aval et disparaît quand l'écoulement principal rejoint la paroi du second conduit. Il est remarquable que très peu de bulles ne soient présentes dans la zone de décollement en aval de l'expansion, ce qui tend à prouver d'une part que les forces moyennes qui agissent sur la migration transversale des bulles (force de portance) sont faibles et que les recirculations moyennes ne sont pas suffisamment énergétiques pour compenser laction des forces de gravité.

L'analyse détaillée des résultats sous forme adimensionnelle prouve que la présence des bulles ne modifie que faiblement la structure de l'écoulement dans la couche de mélange.

\subsection{Analyse des résultats}

\subsubsection{Viscosité turbulente}

Bien que les interactions entre bulles et turbulence relèvent d'une analyse certainement très complexe, il est intéressant de les interpréter en terme de diffusivité turbulente, comme en monophasique, dans la mesure où ce concept garde un sens en raison de la persistance de forts gradients de vitesses et du fort taux de production turbulente dans la zone de mélange en aval de l'élargissement. La viscosité turbulente $v_{L}^{r}$ a été calculée à partir des valeurs expérimentales de la vitesse locale et du cisaillement turbulent

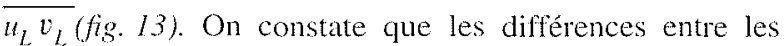
profils adimensionnels de viscosité turbulente avec ou sans bulles restent faibles. Il est instructif d'estimer dans notre cas, la diffusivité induite par les bulles ( $v_{B I}^{t}$ ) introduite par divers auteurs. Selon Serizawa \& Kataoka (1992), par exemple, la modélisation de cette diffusivité est de la forme :

$$
v_{B l}^{\prime}=\frac{1}{3} \alpha \mathrm{d}_{B} u_{L}^{\prime}
$$

Ce terme a été estimé à partir de nos résultats. Il est tracé sous forme adimensionnelle (fig. l3) et comparé avec la viscosité turbulente $v_{L}^{l}$. On constate que sa contribution ne représente au plus qu'environ $10 \%$ de la viscosité turbulence totale et peut de ce fait être négligée en première approximation.

\subsubsection{Bilan radial de quantité de mouvement}

En faisant l'hypothèse que les gradients de pression sont identiques dans chaque phase, le bilan radial de quantité de mouvement peut être écrit sous la forme suivante:

$$
\begin{aligned}
(1-0)( & \left.\overline{U_{L}} \frac{\partial \bar{V}_{L}}{\partial z}+\overline{V_{L}} \frac{\partial \overline{V_{L}}}{\partial r}\right)+(1-\alpha) \times \\
& \left(\frac{\partial v_{L}^{2}}{\partial r}-\frac{\partial \overline{u_{L} v_{L}}}{\partial z}\right)-\left(\overline{v_{L}^{2}} \frac{\partial \alpha}{\partial r}+\overline{u_{L} v_{L}} \frac{\partial \alpha}{\partial z}\right)=M_{L_{r}}
\end{aligned}
$$

Les composantes de vitesses de la phase continue sont notées $\left(U_{L}, V_{L}, W_{L}\right)$ dans le repère de coordonnées cylindriques $(Z, r, \theta)$.

Chacun des termes de cette équation de bilan a été estimé d'après les résultats expérimentaux, excepté le terme de transfert radial de quantité de mouvement $M_{L \text {, }}$

En accord avec la forme généralement acceptée des forces qui s'exercent sur des bulles à grands nombres de Reynolds (Auton et al., 1988 ; Drew and Lahey, 1987 ; Lance, 1991: Rivero, 1991 ; Magnaudet et al., 1994), $M_{I}$ représente la somme des forces de traînée, de masse ajoutée, de portance,... Etant donné la faible contribution de la vitesse moyenne transversale de glissement, seule la force de portance moyenne intervient dans pour traduire le bilan radial de quantité de mouvement $M_{L}$ (Zun (1980); Beyerlein (1985)). Ce terme est de la forme:

$$
M_{L_{r}} \approx-\alpha p_{L} C_{L} U_{R}\left(\frac{\partial \overline{U_{L}}}{\partial r}-\frac{\partial \overline{V_{L}}}{\partial Z}\right)
$$

où $C_{L}$ est le coefficient de portance $\left(C_{L}=0,5\right.$ pour une bulle sphérique).

Le bilan radial de quantité de mouvement déduit de nos mesures prouve que cette force de portance moyenne est trop petite pour réaliser l'équilibre de ce bilan. Une analyse plus réaliste qui introduit non seulement les expressions des forces moyennes mais également les contributions des forces fluctuantes moyennées a été conduite. Elle a montré qu'il est essentiel de prendre en compte les interactions non linéaires des fluctuations des forces agissant sur les bulles.

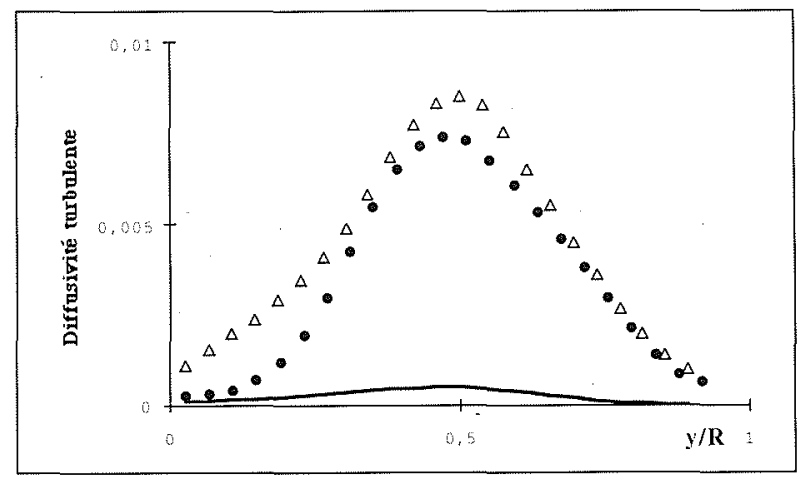

13. Diffusivité turbulente adimensionnelle en monophasique $(\bullet)$, en régime à bulles $(\Delta)$ et modèle de $\mathrm{Se}$ rizawa \& Kataoka (trait continu). 


\section{E CONCLUSION}

Les expériences présentées ici mettent en évidence plusieurs propriétés des écoulements à bulles à faible taux de vide.

La première est la persistance de la structure de l'écoulement monophasique, et en particulier des lois de similitude qui se conservent dans le cas de la couche de mélange et de la couche limite.

La deuxième caractéristique réside dans le rôle joué par les structures cohérentes sur la répartition spatiale des phases par capture des bulles.

La troisième est la modification de la turbulence de la phase liquide. Celle-ci résulte de l'interaction entre les structures turbulentes et le champ de vitesse autour des bulles, ainsi que de la modification des mécanismes de production habituels en monophasique. D'une simple superposition linéaire des effets pour les taux de vide évanescents, cette interaction prend très rapidement un aspect complexe pour des concentrations d'air plus élevées.

Enfin, les transferts de quantité de mouvement entre phases doivent prendre en compte les forces instantanées (telles que portance et traînée), incluant les effets de déformation de l'interface.

Nous remercions le Centre National de la Recherche Scientifique, qui a soutenu ces actions au travers du GDR 1027, et la CEE pour son soutien via le programme Capital Humain et Mobility.

\section{Références}

[1] Serizawa A., Kataoka I. and Michiyosh 1. (1975). "Turbulence structure of air-water bubbly flows. Part Il : local properties". Int. J. Mulniphase Flow; 2, 235-246.

[2] WANG L.P. and MAXEY M.R. (1993). - "Settling velocity and concentration distribution of heavy particles in homogeneous turbulence". J. of Fluid Mech., 256, 27-68.

[3] Lazaro B.J. and Lasheras J.C. (1989). - "Particle dispersion in a turbulent, plane shear layer". Phys. Fluids A, 1, 1035.

14] SQuires K.D. and EATON J.K. (1991). - "Preferential concentration of particles by turbulence". Phys. Fluids A, 3, 1169

[5] Lance M., Marie J.L. and Batallle J. (1991). - "Homogeneous Turbulence in Bubbly Flows". J. Fluids Engineering, 113, pp. 295-300.

[6] RoIG V. (1993). - Thèse de Doctorat, Institut de Mécanique des Fluides de Toulouse.

[7] Moursali E., Marié J.L. and Batallee J. (1995). - "An upward turbulent bubbly boundary layer along a vertical fat plate". Int. J. Multiphase Flow: To be published.

[8] Bel Fohila R., Suzanne C. and Masbernat L. (1992). "Two-Phase Bubbly Flow Measurements in a Vertical Sudden Area Expansion", in 6th Workshop on Two-Phase Flow Predictions, 30 March-2 April, Erlangen, Germany, pp. 1392-1401.

[9] Champagne F.H., Harris V.G. and Corrsin S. (1970). "Experiments on nearly homogeneous shear flows". J. of Fluid Mech., 41, 81-139.

[10] Rajaratnam N. (1976). - "Turbulent jets". Elsevier Scientific Publishing Company.
[11] LANCE M. and BATAille J. (1991). - "Turbulence in the liquid phase of a uniform bubbly air-water flow". J. Fluid Mech., 222, 95-118.

[12] Serizawa A., Kataoka I., Zun I. and Michyoshi I. (1988). - "Bubble size effect on phase distribution". In Proc: of the Japan-US Seminar on Two-Phase Flow Dynamics, pp. 15-20, Ohtsu, Japan.

[13] Monj H. and Matsui G. (1991). - "Effect of bubble size on structure of vertical bubble flow". In Proc. of the Int. Conf. on Multiphase Flows, pp. 449-452, Tsukuba.

[14] Liu T.J. (1993). - "Bubble size and entrance length effects on void development in a vertical channel". Int. J. Multiphase Flow, 19, 99-113.

[15] Zun 1., Kl.jenak I. and Serizawa A. (1992). - "Bubble coalescence and transition from wall void peaking to core void peaking in turbulent bubbly flow". In Dynamics of Two-Phase Flows (Edited by Jones, O.C. \& Michiyoshi, I.), pp. 233-249. Boca Raton, FL.

[16] BLACKWELder R.F. and HARITONIDIS J.H. (1983). - "Scaling of the bursting frequency in turbulent boundary layers". J. Fluid Mech. 132, 87-103.

[17] WANG S.K., Lee S.J., JONES O.C. and LAHEY R.T. (1987) - "3D Turbulence structure and phase distribution measurements in bubbly two-phase flows". Int. J. Multiphase Flow, 13, 327-343.

[18] Serizawa A. and Kataoka I. (1992). - "Dispersed Flow" Third International Workshop on Two-Phase Flow Fundamentals, Imperial College, London, June 15th-19th.

[19] Auton T.R., Hunt J.C.R. and Prud Homme M. (1988). "The force exerted on a body in inviscid unsteady nonuniform rotational flow". J. Fluid Mech., 197, 197-241.

[20] DREW D.A. and LAHEY R.T. (1987). — "The virtual mass and lift force on a sphere in rotating and straining inviscid flow", Int. J. Multiphase Flow, 13, $\mathrm{N}^{\circ} 1$, pp. 113-121.

[21] Rivero M., Magnaudet J. et Fabre J. (1991). - "Quelques résultats nouveaux concernant les forces exercées sur une inclusion sphérique par un écoulement accéléré", C.R. Acad. Sci. Paris, t. 312, Série 1l, p. 1499-1506.

[22] Magnaudet J., Rivero M., Fabre J. (1994). - "Accelerated flow past a rigid sphere or spherical bubble. Part I-Steady straining Flow", J. of Fluid Mech, 284, 97-135.

[23] ZUN I. (1980). - "Transverse migration of bubbles influenced by walls in vertical bubbly channel", Int. J. Multiphase Flow, 6, p. 583.

[24] Beyerlein S.W., Cossman R.K. and Richter H.J. (1985). - "Prediction of Bubble Concentration Profiles in Vertical Two-Phase Flow", Int. J. Multiphase Flow, 11, pp. 629-649.

[25] Lance M. and Lopez De Bertodano M. (1992). - "Phase distribution and wall effects in bubbly flows", $3 \mathrm{rd}$ International Workshop on Two-Phase Flow's Fundamentals, London, June 15-19.

[26] Moursali E. (1993). - «Etude expérimentale d'une couche limite eau-air à bulles se développant sur une plaque plane verticale». PhD. Thesis, Ecole Centrale de Lyon, Ecully, France.

[27] Theofanous T.G. and Sullivan J. (1982). - "Turbulence in two-phase dispersed flows". J. Fluid Mech., 116, 343.

[28] DREW D.A: and LAHEY R.T. (1982). - "Phase distribution mechanisms in turbulent low-quality two-phase flow in a circular pipe". J. Fluid Mech, 117, 91-106.

[29] BEL FDHLA R. (1991). - "Analyse expérimentale et modélisation d'un écoulement vertical à bulles dàns un élargissement brusque". Thèse de Doctorat, I.M.F.T., Toulouse. 